\title{
Mechanical modeling of the bandgap response of tensegrity metamaterials
}

Cite as: AIP Conference Proceedings 2116, 260010 (2019); https://doi.org/10.1063/1.5114261

Published Online: 24 July 2019

Ada Amendola, Anastasiia Krushynska, Mariella De Piano, Chiara Daraio, Nicola M. Pugno, and Fernando Fraternali

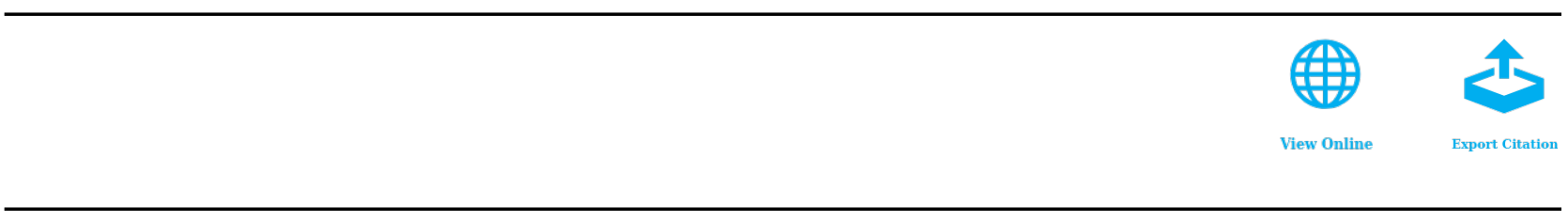

\section{Conference Proceedings}

Get $30 \%$ off all print proceedingis! 


\title{
Mechanical Modeling of the Bandgap Response of Tensegrity Metamaterials
}

\author{
Ada Amendola ${ }^{1}$ a) , Anastasiia Krushynska ${ }^{2}$ b) , Mariella De Piano ${ }^{1 \text { c) }}$, \\ Chiara Daraio $^{3 \mathrm{~d})}$, Nicola M. Pugno ${ }^{4 \mathrm{e}}$, Fernando Fraternali ${ }^{1 \mathrm{f}}{ }$ \\ ${ }^{1}$ Department of Civil Engineering, University of Salerno, Via Giovanni Paolo II, 132, 84084 \\ Fisciano (SA), Italy. \\ ${ }^{2}$ Department of Physics, University of Turin, Via P. Guria, 1, 10125 Turin, Italy, \\ ${ }^{3}$ Engineering and Applied Science, California Institute of Technology \\ Pasadena, CA 91125, USA. \\ ${ }^{4}$ Laboratory of Bio-Inspired and Graphene Nanomechanics, Department of Civil, \\ Environmental and Mechanical Engineering, University of Trento, 38123 Trento, Italy. \\ a)adaamendola1@unisa.it, b)akrushynska@gmail.com, ${ }^{\mathrm{c}}$ mdepiano@unisa.it, d)daraio@caltech.edu \\ e)nicola.pugno@unitn.it, ${ }^{\mathrm{f}}$ Corresponding author: f.fraternali@unisa.it
}

\begin{abstract}
We investigate the acoustic band structure of tensegrity mass-spring chains as a function of the applied, local and global states of prestress. We first study the bandgap response of linear monoatomic chains, which show lumped masses connected to tensegrity prisms acting as mechanical springs. Next, we present a numerical study on the nonlinear wave dynamics of the examined systems in the geometrically nonlinear regime that is induced by the presence of moderately large relative displacements between the lumped masses. Such a study confirms the results of the linear analysis in the case of an elastically hardening system.
\end{abstract}

\section{INTRODUCTION}

In the last few years, lattice metamaterials are becoming popular in different areas of research because of their peculiar, mechanical and acoustic behaviors [1-6]. The present work investigates the dynamics of a $1 \mathrm{~d}$ tensegrity metamaterials [7-9], and it is aimed at characterizing the acoustic band structure of such systems as a function of the applied, local and global prestress [4]. The first part of this paper provides an analytic study of the bandgap response of linear monoatomic chains, which are formed by lumped masses connected to linear springs consisting of tensegrity prisms. The response of such systems can be effectively tuned by pre-stretching the cables forming the individual units (internal self-stress), and/or by applying global compression forces to the terminal bases of the chain (global prestress), while keeping material properties unaltered [7]. The second part of the paper deals with a numerical study on the nonlinear wave dynamics of monatomic chains under moderately large displacements. Such a study proves that elastically hardening systems exhibit frequency band gaps also in the geometrically nonlinear regime.

\section{DISPERSION RELATION OF 1D TENSEGRITY CHAINS}

Let us investigate the tuning of the dispersion relation of tensegrity chains formed by tensegrity units alternating massive discs, modeled by linear elastic springs and lumped masses [4]. The elastic springs are considered of the same value of the stiffness constant k (Fig. 1). We define $\delta$ as the axial displacement from the reference configuration $\left(\delta=\bar{h}-\mathrm{h}_{0}\right)$ and we consider $\varepsilon=\delta / \bar{h}$ as the axial strain, positive if the structure is compressed.

We focus our study on the behavior of a first chain formed by prisms with a non-linear stiffening response in terms of $\mathrm{F}-\varepsilon$ ('Mono 1') and a second chain where the prisms forming the structure are characterized by a softening response ('Mono 2'). The F - $\varepsilon$ curves exhibited by the systems under consideration is shown in Fig. 2 . The initial configuration of each system is marked by circles in Fig. 2.

International Conference of Numerical Analysis and Applied Mathematics (ICNAAM 2018)

AIP Conf. Proc. 2116, 260010-1-260010-4; https://doi.org/10.1063/1.5114261

Published by AIP Publishing. 978-0-7354-1854-7/\$30.00 
In this section, we linearize such a non-linear response near the initial configuration, to examine infinitesimally small oscillations of the chains Mono 1 and Mono 2.

Note that the tangent stiffness $\mathrm{k}$ of the first System is relatively low (Fig. $2 \mathrm{a}: \mathrm{F}_{0}=\mathrm{F}_{01}=7.31 \mathrm{~N}, \varepsilon_{0}=\varepsilon_{01}=$ $0.010, \mathrm{k}=\mathrm{k}_{1}=394.89 \mathrm{~N} / \mathrm{m}$ ) compared to the one of Mono $2\left(\right.$ Fig. $2 \mathrm{~b}: \mathrm{F}_{0}=\mathrm{F}_{02}=36.14 \mathrm{~N}, \varepsilon_{0}=\varepsilon_{02}=0.199, \mathrm{k}=\mathrm{k}_{2}$ $=2165.30 \mathrm{~N} / \mathrm{m})$.

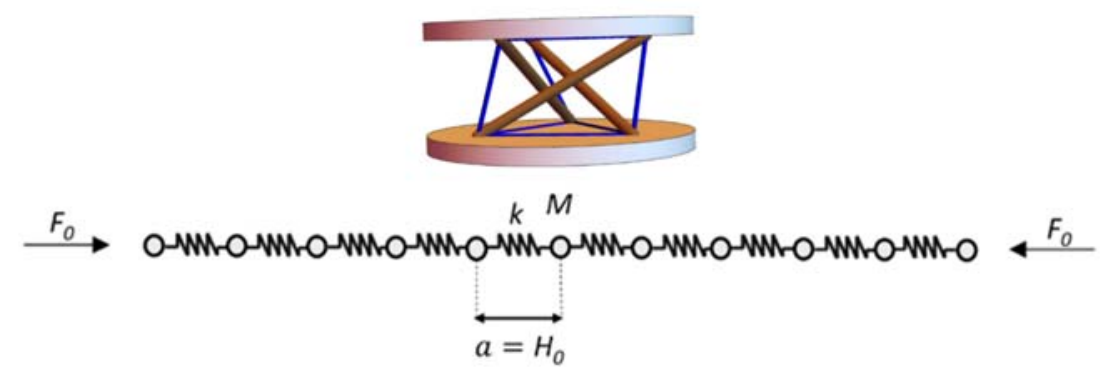

FIGURE 1. Monoatomic chain: geometry of the unit cell (top) and mass-spring model (bottom).

It is worth noting that the tangent stiffness $\mathrm{k}$ at the equilibrium point is equal to the slope of the local tangent line to the $\mathrm{F}-\varepsilon$ curve multiplied by the equilibrium height $\bar{h}$ (Fig. 2).

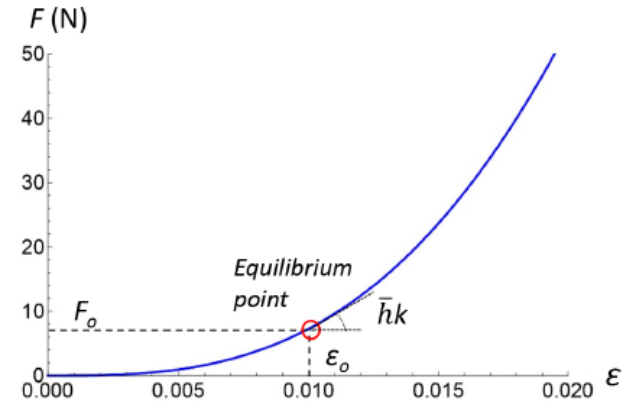

(a)

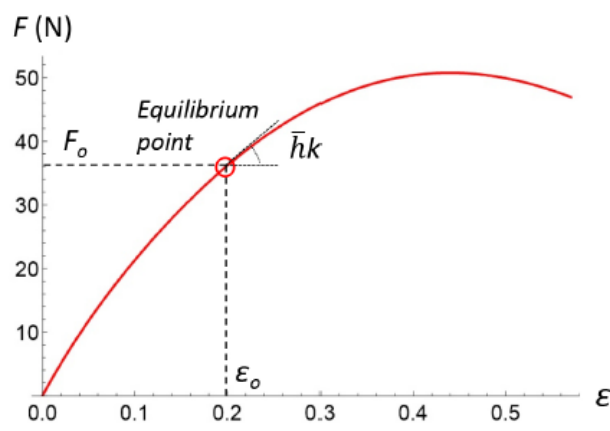

(b)

FIGURE 2. $\mathrm{F}$ - $\varepsilon$ curves of systems Mono 1 (a), and Mono 2 (b).

By referring to the Bloch-Floquet theory given in Refs. 10 for one-dimensional periodic systems, we easily obtain a single dispersion curve (the so-called 'acoustic branch') for the Mono 1 and Mono 2 systems, which shows that the frequency bandgap region of such systems is easily tunable by adjusting the local and global states of prestress applied to the structure, while leaving material properties unchanged (see Figure 4 of Ref. [7])

\section{FREQUENCY BAND GAPS UNDER MODERATELY LARGE INCREMENTAL STRAINS}

In the present section we investigate on the phenomenon of wave attenuation in the Mono 1 system, by considering the non-linear response under moderately large incremental strains from the equilibrium configuration. This study was conducted numerically, by applying a sinusoidal time-displacement input to the first unit of a chain composed of 100 masses to perturb the equilibrium configuration.

Fig. 4 illustrates the fast Fourier transforms (FFTs) of the outputs for units 1 and 50 at excitation frequencies ranging between $20 \mathrm{~Hz}$ and $80 \mathrm{~Hz}$. The results illustrated in such a figure highlight that disturbances with input frequencies above $40 \mathrm{~Hz}$ are dramatically attenuated during their propagation through the system. 

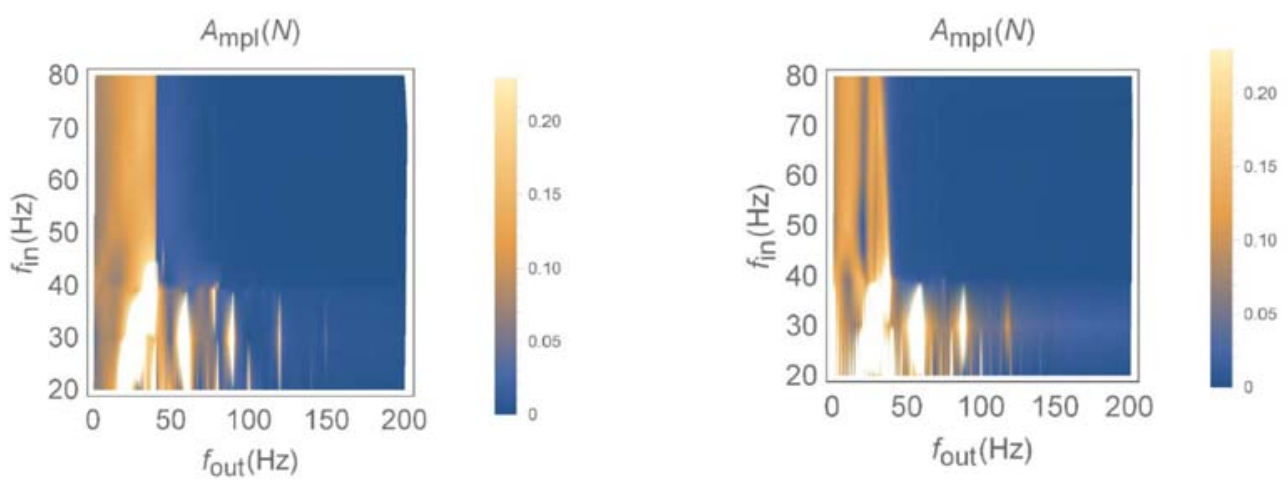

FIGURE 3. FFTs of the outputs for unit \# 5 (left) and unit \# 50 (right) for excitation frequencies ranging from 20 to $80 \mathrm{~Hz}$.

\section{CONCLUDING REMARKS}

We have presented a theoretical and numerical study on monoatomic mass-spring chains, which are formed by alternating tensegrity prisms with massive disks. The given results have shown that the examined structures exhibit highly tunable frequency band gaps in the linear and non-linear regimes induced by small or moderately large vibration near the initial equilibrium state, as a function of the applied global and local states of prestress. Future studies will investigate engineering applications of $2 \mathrm{~d}$ and $3 \mathrm{~d}$ versions of the systems examined in the present work [11-13], the insertion of curved struts within the tensegrity units [14-15], and the fabrication and testing of physical models of the examined structures through sustainable additive manufacturing techniques [16$17]$.

\section{REFERENCES}

1. F. Fraternali, F., L. Senatore, C., Daraio, "Solitary waves on tensegrity lattices”, J Mech Phys Solids 60, 1137-1144 (2012).

2. D. Ngo, F. Fraternali, C. Daraio, "Highly Nonlinear Solitary Wave Propagation in Y-Shaped Granular Crystals with Variable Branch Angles". Phys Rev E 85, 036602-1-10 (2012).

3. F. Fraternali, A. Amendola, "Mechanical modeling of innovative metamaterials alternating pentamode lattices and confinement plates". J Mech Phys Solids 99, 259-271 (2017).

4. F. Fraternali, G. Carpentieri, A. Amendola, R.E. Skelton, and V.F. Nesterenko, "Multiscale tunability of solitary wave dynamics in tensegrity metamaterials". Appl. Phys. Lett. 105, 201903 (2014).

5. C. Davini, A. Micheletti, P. Podio-Guidugli, "On the impulsive dynamics of T3 tensegrity chains". Meccanica 51(11), 2763-2776 (2016).

6. A. Krushynska, A.Amendola, F. Bosia, C. Daraio, N.M. Pugno, F. Fraternali, “Accordion-like metamaterials with tunable ultra-wide low-frequency band gaps". New J. Phys., Online First, https://doi.org/10.1088/13672630/aad354 (2018).

7. A. Amendola, A. Krushynska, C. Daraio, N.M. Pugno, F. Fraternali, "Tuning frequency band gaps of tensegrity metamaterials with local and global prestress". Int. J. Solids Struct., Online First https://doi.org/10.1016/j.ijsolstr.2018.07.002 (2018).

8. R. Montuori, R.E. Skelton, "Globally stable tensegrity compressive structures for arbitrary complexity". Comp. Struct. 179, pp. 682-694 (2017).

9. R.E. Skelton, R. Montuori, V. Pecoraro, "Globally stable minimal mass compressive tensegrity structures". Comp. Struct. 141, pp. 346-354 (2016).

10. E. B.Herbold, J. Kim, V. F. Nesterenko, S. Y. Wang, C. Daraio, "Pulse propagation in a linear and nonlinear diatomic periodic chain: effects of acoustic frequency band-gap". Acta Mech 205 (2009).

11. F. Fraternali, C.D. Lorenz, G. Marcelli, "On the estimation of the curvatures and bending rigidity of membrane networks via a local maximum-entropy approach". J. Comput. Phys. 231, 528-540 (2012).

12. F. Fraternali, J.N. Reddy, “A penalty model for the analysis of laminated composite shells”. Int. J. Solids Struct. 30, 3337-3355 (1993).

13. B. Schmidt, F. Fraternali, "Universal formulae for the limiting elastic energy of membrane networks". J. Mech. Phys. Solids 60, 172-180 (2012). 
14. L. Ascione, F. Fraternali, "A penalty model for the analysis of curved composite curved beams". Comput. Struct. 45, 985-999 (1992).

15. F. Fraternali, G. Bilotti, "Nonlinear elastic stress analysis in curved composite beams". Comput. Struct. 62, 837-869 (1997).

16. R. Singh, R. Kumar, N. Ranjan, R. Penna, F. Fraternali, "On the recyclability of polyamide for sustainable composite structures in civil engineering". Compos. Struct., 184, 704-713 (2018).

17. R. Singh, S. Singh, F. Fraternali, "Development of in-house composite wire based feed stock filaments of fused deposition modelling for wear-resistant materials and structures". Comp. Part B: Eng 98, 244-249 (2016). 